\title{
Cherry Picking
}

\author{
Wenfeng Qiu*and Megan Lang ${ }^{\dagger}$
}

August 18, 2021

\begin{abstract}
Measures like pre-analysis plans ask researchers to describe planned data collection and justify data exclusions, but they provide little enforceable oversight of primary data collection. We show that a simple algorithm can select large subsets of data that yield economically meaningful and statistically significant treatment effects. The subsets cannot be distinguished from a random sample of the original data, rendering the selection undetectable if peer reviewers are unaware of the size of the original dataset. Our results hold using simulated data and replication data from a well-known study. We show that there are few natural deterrents to dataset manipulation: the results in our selected subset are robust to a range of alternative specifications, our algorithm performs well under complex sampling strategies, and our subset can yield artificially high effects on multiple outcomes. We conclude by proposing a measure to prevent such manipulation in field experiments.
\end{abstract}

JEL Codes: C81, C83, C93

\footnotetext{
*University of California Berkeley Department of Agricultural and Resource Economics. wqiu03@berkeley.edu.

${ }^{\dagger}$ King Climate Action Initiative and University of California Santa Barbara. meganlang@ucsb.edu Thanks to Ethan Ligon, Jeremy Magruder, Edward Miguel, Karl Dunkle Werner, and Fernando Hoces de la Guardia for helpful comments.
} 


\section{Introduction}

Growing concerns about the reliability of empirical research have led scholars to develop best practices for transparent, reproducible research (e.g., Ofosu and Posner (2021), Lenz and Sahn (2021), Miguel et al. (2014), LeBel et al. (2013), Stroebe, Postmes, and Spears (2012), Simmons, Nelson, and Simonsohn (2011)). The goal of these practices is generally to limit researcher degrees of freedom to prevent positive selection of results that are statistically significant. Within economics, it is now common for researchers collecting primary data or conducting a randomized control trial (RCT) to pre-register their studies and to provide access to their data upon publication. The set of norms and institutions around research transparency are well-suited to addressing "non-fraudulent" concerns with empirical research. However, multiple scholars have acknowledged that current practices can do little to prevent and detect fraud (LeBel et al. (2013), Simmons, Nelson, and Simonsohn (2011)). Given the time and resources that researchers invest in field experiments and continued perceptions that null results are difficult to publish well, unscrupulous researchers face incentives to manipulate results.

In this paper, we demonstrate that an unscrupulous researcher faced with a null result when estimating an average treatment effect (ATE) could employ a simple, flexible algorithm to select a subset of data that yields a statistically significant ATE an order of magnitude larger than the true effect. Even subsets as large as $95 \%$ of the original sample may generate ATEs which are large and statistically significant. We further demonstrate that a typical peer reviewer or outside observer who is unaware of the size of the full dataset cannot detect the use of the algorithm ex post, even if they have access to data from the population from which the sample is selected. We go on to show that our algorithm works using real world data, and continues to yield large and significant results under a range of real world complexities and robustness checks that a researcher may be asked to undertake during peer review. ${ }^{1}$ Finally, we demonstrate that applying the algorithm for one outcome may yield positive ATEs on a number of other outcomes even when they are only moderately correlated. Combined, our results suggest that there are few natural deterrents to this type of unethical behavior.

We are not the first to point out that unscrupulous researchers may manipulate data and avoid detection. Stroebe, Postmes, and Spears (2012) review a sample of forty cases of researcher fraud across medical and social sciences and conclude that peer review and replication are unlikely to detect, and therefore to prevent, instances of fraud. They argue that this is particularly true in social psychology because there are a host of factors that can ex-

\footnotetext{
${ }^{1}$ The one exception is the robustness measure proposed in Broderick, Giordano, and Meager (2021), which we discuss in detail.
} 
plain non-replication, an issue likely faced by many social science experiments. Furthermore, cases where fraud have been detected in the past are overwhelmingly due to whistleblowers coming forward with inside information.

While we agree with the broad conclusion of Stroebe, Postmes, and Spears (2012), our primary contribution is to demonstrate that an unscrupulous researcher does not need to fabricate data to ensure they find theoretically grounded, statistically significant results. Our finding is important for fields like development economics and experimental economics, where large research teams are often employed to collect data for principal investigators. Complete data fabrication is exceedingly unlikely in these fields, but we show that unscrupulous researchers can guarantee large and statistically significant results by strategically selecting large subsets of empirical datasets.

Similarly, many reporting standards (or proposed standards) for journals require that researchers justify all data exclusions (Miguel et al. (2014), LeBel et al. (2013), Stroebe, Postmes, and Spears (2012)). We show that an unscrupulous researcher using primary data could employ an algorithm like ours to select a single subset of data that they use to generate all of the results in a journal article. They could go so far as to share the entire subset of data to comply with journal requirements. Without mandatory disclosure of the size of the originally collected dataset, it is impossible for peer reviewers or other researchers to detect that the full primary dataset is actually larger than the one used to estimate the results.

Two recent papers also study how selecting subsets of the data can affect estimations and inferences, although their primary goals are to raise concerns for robustness checks. In a study of 31 published papers, Young (2020) shows that statistically significant results from IV regressions generally depend on only one or two observations or clusters. Hence, removing these data points will substantially pivot the main findings of the papers. Broderick, Giordano, and Meager (2021) propose an approach to find the fraction of the data with the approximately maximal influence on the results. They demonstrate how this approach can be used for robustness checks and find e.g., in a microcredit study by Angelucci, Karlan, and Zinman (2015), the sign of the ATE could be alternated by removing a single data point. Their approach can also be employed for cherry picking subsets of data and generally yields even higher ATEs than our approach. The key difference between their approach and ours is that theirs merely focuses on finding the maximal influential subset while ours require the selected subset to be representative of the original data. In an RCT scenario, the covariates of the treated and control groups may not be balanced in the subset selected by their approach. $^{2}$ Thus, our approach can also be employed as a less stringent robustness check.

\footnotetext{
${ }^{2}$ Technically speaking, if there is only one data point removed then the remaining data are very likely to be representative of the original data, regardless of the selection criteria. However, we focus on what we
} 
The remainder of the paper is structured as follows. In section 2, we demonstrate the efficacy of our algorithm for selecting data for positive ATEs using simulated data. We further show that the algorithm can flexibly accommodate multiple outcomes. In section 3, we apply the algorithm to real world data from Banerjee et al. (2015). We reproduce the results in the original paper, then go on to show that using the algorithm to select a $95 \%$ subsample of the data yields positive and statistically significant ATEs on a range of outcomes. We demonstrate that the algorithm continues to perform well under a range of sampling strategies and robustness checks, and that even without optimizing the algorithm to generate positive ATEs on multiple outcomes, selecting a subsample based on total consumption yields positive and statistically significant impacts on a range of business outcomes. In section 4, we discuss the implications for research transparency and conclude by proposing a data monitoring institution capable of preventing researchers from cherry picking observations.

\section{$2 \quad$ Cherry Picking with Simulated Data}

For simplicity, we restrict our attention to RCT settings in this paper but the idea can be extended to any setting when linear regressions are used for causal inference. We say the cherry picking is undetectable if the selected subset is representative of the original data, that is,

1. The RCT structure is preserved so the treatment assignment in the selected subset is still randomized with respect to all covariates.

2. The probability distributions of the covariates in the selected subset are the same as those in the original data.

The first requirement to preserve the RCT structure is necessary since otherwise the RCT design would be unreliable. The set of covariates will often contain variables that are publicly available, e.g., individual's age. If the sample distribution of age in a study is very different from the corrosponding population distribution, then people might be suspicious of the study. The second requirement addresses such concerns. Throughout the paper, we will conduct various statistical tests to show our selected subset is indeed satisfying these two requirements and is hence undetectable.

view as more typical cases where a researcher would need to remove a large enough subset of the data that covariates may become unbalanced. 


\subsection{Cherry Pick for A Single Outcome}

We start with a simulated example to illustrate our cherry picking algorithm and to demonstrate its efficacy. Consider the following data generating process

$$
y=0.1+0.3 x_{1}-0.2 x_{2}+0 \cdot d+\epsilon
$$

where $y$ is the outcome variable of interest, $d$ (or "treat") is the randomized treatment assignment and $x_{1}, x_{2}$ are correlated observable covariates that are potentially correlated with the unobservable error term $\epsilon$. From the data generating process, there is no treatment effect for $y$. In the simulation, there are $N=2000$ observations. The treatment assignment is randomized with around $30 \%$ of the observations being treated and $x_{1}, x_{2}, \epsilon$ are generated from multivariate normal distributions with zero means and unitary variances (see Table A1 in the appendix for a summary of the simulated data and the covariates balance table).

We run linear regressions, using all of the 2000 observations, to estimate the average treatment effects. The results are summarized in the first two columns in Table 1. As expected, the estimated effects are close to zero. We will use our algorithm to cherry pick a subset containing 1500 observations from the original data. Our goal is to obtain a positive and statistically significant ATE when we do the same regression analysis using the cherry picked subset.

Our cherry picking is based on each observation's score, as we pick the observations with highest scores. The scores are calculated by our algorithm as follows:

- Step 0: Each observation $i$ 's score is initialized to be $\operatorname{scor} e_{i}^{0}=0$. Denote $\tau_{0}$ as the estimated treatment effect from the regression using all observations with all covariates. We will do the following steps $\mathbf{1 , 2}$ repeatedly for $K$ rounds (e.g., $K=500$ ).

- Step 1: In round $k$, randomly draw a sample $S_{k}$ from the original data containing $N_{k}$ (e.g., $N_{k}=N / 2=1000$ ) observations without replacement. Run a regression with all covariates using the sample $S_{k}$ and denote the estimated treatment effect as $\tau_{k}$.

- Step 2: For any observation $i$ in sample $S_{k}$, update its score by adding $\left(\tau_{k}-\tau_{0}\right)$ so score ${ }_{i}^{k} \longleftarrow$ score $_{i}^{k-1}+\left(\tau_{k}-\tau_{0}\right)$ if $j \in S_{k} ;{ }^{3}$ for any observation $j$ not in sample $S_{k}$, its score is unchanged so score $e_{j}^{k} \longleftarrow$ score $e_{j}^{k-1}$ if $j \notin S_{k}$.

Once we obtain the scores, we pick 1500 observations with the highest scores. We do the same regression analysis as before. The results are shown in the last two columns of Table 1.

\footnotetext{
${ }^{3}$ If $\tau_{0}$ is not statistically significant from zero, we can optionally replace $\tau_{0}$ with zero.
} 
Table 1: Linear Regressions Before/After Cherry Picking

\begin{tabular}{|c|c|c|c|c|}
\hline & \multicolumn{4}{|c|}{ Dependent variable: } \\
\hline & \multicolumn{4}{|c|}{$\mathrm{y}$} \\
\hline & (1) & $(2)$ & (3) & (4) \\
\hline treat & $\begin{array}{c}0.075 \\
(0.048)\end{array}$ & $\begin{array}{l}0.074^{*} \\
(0.043)\end{array}$ & $\begin{array}{c}0.252^{* * *} \\
(0.054)\end{array}$ & $\begin{array}{c}0.254^{* * *} \\
(0.049)\end{array}$ \\
\hline $\mathrm{x} 1$ & & $\begin{array}{c}-0.127^{* * *} \\
(0.028)\end{array}$ & & $\begin{array}{c}-0.111^{* * *} \\
(0.031)\end{array}$ \\
\hline $\mathrm{x} 2$ & & $\begin{array}{c}-0.318^{* * *} \\
(0.028)\end{array}$ & & $\begin{array}{c}-0.330^{* * *} \\
(0.031)\end{array}$ \\
\hline Constant & $\begin{array}{c}0.092^{* * *} \\
(0.026)\end{array}$ & $\begin{array}{c}0.099^{* * *} \\
(0.023)\end{array}$ & $\begin{array}{c}0.037 \\
(0.029)\end{array}$ & $\begin{array}{l}0.046^{*} \\
(0.027)\end{array}$ \\
\hline Observations & 2,000 & 2,000 & 1,500 & 1,500 \\
\hline $\mathrm{R}^{2}$ & 0.001 & 0.175 & 0.014 & 0.186 \\
\hline
\end{tabular}

Using the cherry picked data, we obtain statistically significant ATEs and the effects are very large, given the standard deviation of $y$ is close to 1 . We verify that our cherry picked subset is representative of the original data. The correlation between the scores and the covariates are close to zero, which suggests the cherry picking does not affect the distributions of the covariates. We conducted several two-sample Kolmogorov-Smirnov tests for (1) covariates in the cherry picked data/original data and (2) covariates in the treatment/control group in the cherry picked data. The p-values are all close to 1, indicating that the summary statistics for all covariates in the cherry picked data are similar with those in the original data. ${ }^{4}$ Moreover, the coviarates are still balanced with respect to the treatment assignment, suggesting that the treatment assignment in the picked data is still randomized. In fact, the treatment assignment is not random because it is positively correlated with the unobservable $\epsilon$, but this can never be detected since $\epsilon$ is unobservable.

It is worthwhile to discuss the intuition behind our algorithm. Since there is no true treatment effect in the data generating process, the estimation using the cherry picked data must be upward biased. In each round of the algorithm, $\left(\tau_{k}-\tau_{0}\right)$ is the bias that comes from

\footnotetext{
${ }^{4}$ See Table A2 in the appendix, where we present a summary of the cherry picked data that is analogous to Table A1.
} 
random sampling. The observations with high scores are those contributing to the upward bias by generating positive correlation between the treatment assignment and the error term. The cherry picked subset is representative of the original data since we have controlled for the covariates in the regressions when we calculate the scores.

Figure 1: Estimated Treatment Effects and Percent of Cherry Picked Data

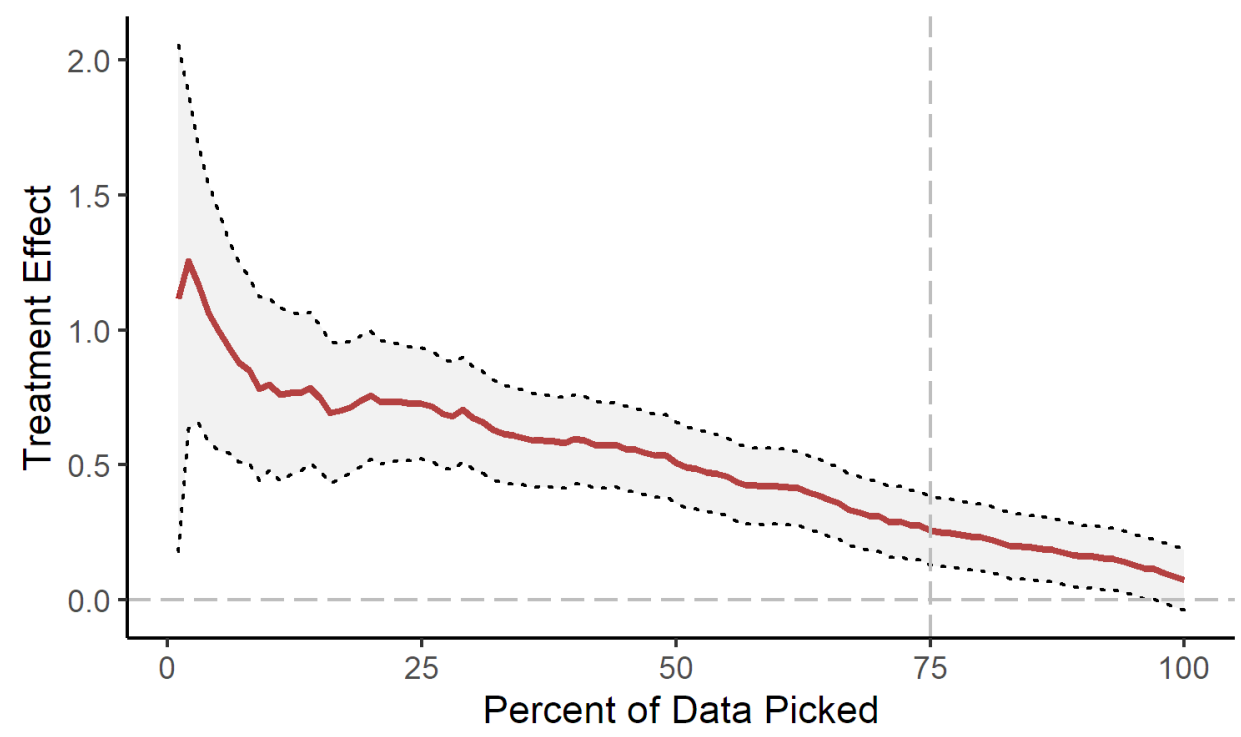

Note: The solid red curve plots the relationship between the percent of data picked and the estimated treatment effect. The dotted curves indicate $99 \%$ confidence intervals. The horizontal dashed line indicates the level of zero treatment effect and the vertical dashed line indicates the level of picking $75 \%$ (1500 out of 2000) of the data.

To visualize the performance of our cherry picking, we present a plot of the relationship between the percent of observations picked and the estimated treatment effect in Figure 1. It is clear that if we have the freedom to remove more observations, then we will obtain larger positive ATEs. The plot suggests that we can remove as few as $3 \%$ of the observations to get a statistically significant treatment effect equal to 0.11 .

Broderick, Giordano, and Meager (2021) develop an approach to select a subset that has approximately maximal influence on the results. Their approach is primarily for robustness checks, as it calculates the amount of data that would need to be removed to change the result. In Figure 2, we provide a visualization of how our cherry picking algorithm performs against their robustness check. There are three robustness measures. The most stringent one is plotted in solid red, which measures the percent of the data required to be dropped to make the estimated treatment effect statistically insignificant. For example, our 95\% cherry picked subset is not robust to their most stringent robustness check at the level of dropping $1 \%$ of the data. But our $75 \%$ cherry picked subset is very robust since at least $4 \%$ 
of the data needs to be dropped to change the significance of the result. As we remove more data in cherry picking, we obtain a higher estimated ATE and our cherry picking becomes increasingly robust to any of their three measures.

Figure 2: Robustness Check by Broderick, Giordano, and Meager (2021)

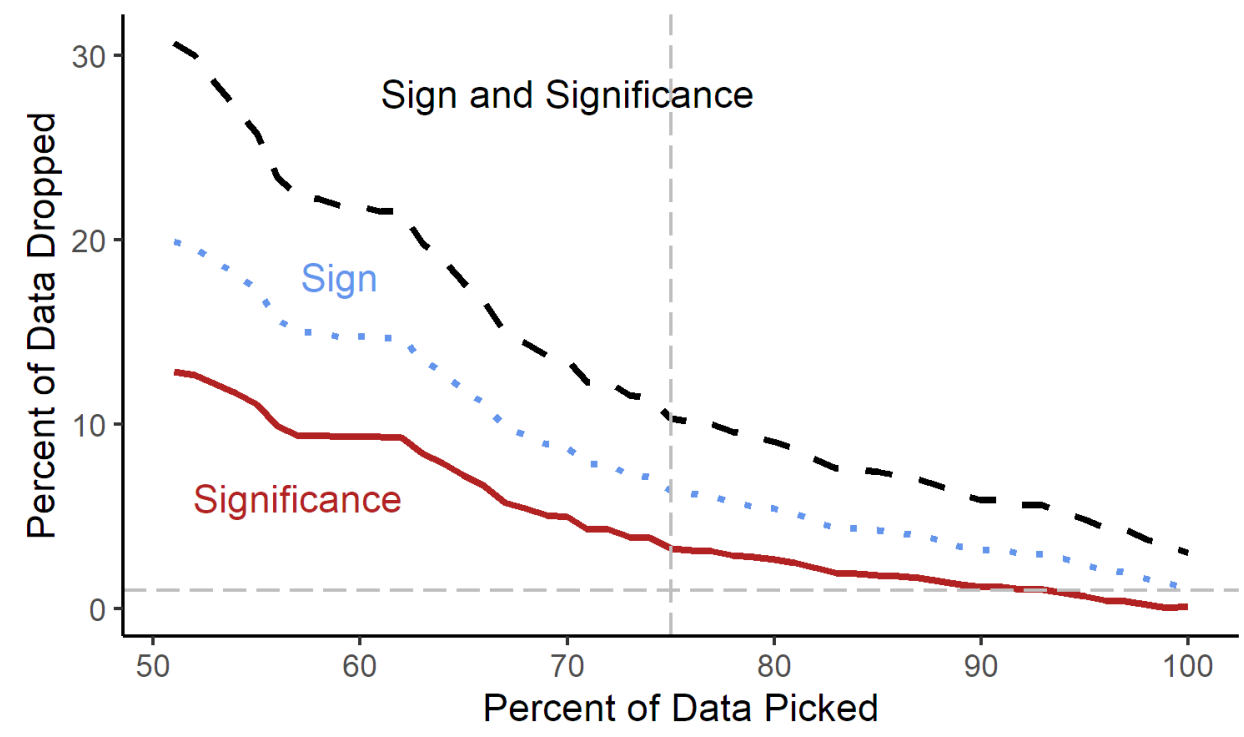

Note: Each robustness measure shows the percent of the data required to be dropped to change the estimated treatment effect. The solid red curve is for eliminating statistical significance, the dotted blue curve is for getting opposite signs, and the black dashed curve is for getting opposite signs with statistical significance. The horizontal dashed line indicates the level of $1 \%$ of the data being dropped and the vertical dashed line indicates the level of picking $75 \%$ (1500 out of 2000) of the data.

From the figure, if we want our cherry picked subset to pass their robustness checks at the level of dropping $\alpha \%$ of the data, then we need to remove more than $\alpha \%$ of the data in our cherry picking. Their selection is based on calculated gradients, which is logically similar to selection based on scores in our algorithm. There are two primary reasons why their selection should have a larger influence on the estimated treatment effects than our cherry picking. First, their selection is approximately maximal influential, while we do not claim our cherry picking to be optimal in any sense. ${ }^{5}$ Second, they do not require the selection to be representative of the original data. This additional requirement further constrains the performance of our cherry picking.

\footnotetext{
${ }^{5}$ We are aware of another way of cherry picking that outperforms our approach in a simple case without any covariate.
} 


\subsection{Cherry Pick for Multiple Outcomes}

So far we have discussed cherry picking for one outcome variable. In reality, many research projects study treatment effects on multiple outcome variables. These outcome variables are typically correlated with each other. In this section, we will show how cherry picking for one outcome variable affects the estimations for other outcome variables, and will explore cherry picking for multiple outcomes.

Consider the following data generating process

$$
\begin{aligned}
& y=0.1+0.3 x_{1}-0.2 x_{2}+0 \cdot d+\epsilon \\
& z=-0.3-0.1 x_{1}+0.5 x_{2}+0.2 \cdot d+\eta \\
& w=0.2-0.4 x_{1}-0.3 x_{2}-0.1 \cdot d+\gamma
\end{aligned}
$$

Now we have three outcome variables $y, z, w$. In particular, the data generating process for $y$ is the same as the previous section. We let $\epsilon, \eta$ be positively correlated and $\epsilon, \gamma$ be negatively correlated (see Table A1 for a summary of the simulated data and Table A3 for regression results for $y, z$, and $w$ using all 2000 observations).

To see how cherry picking for $y$ affects estimations of treatment effects on $z, w$, we do regression analysis using the $75 \%$ cherry picked data, which is the same subset we used in the previous section. We find that the estimated treatment effect for $z$ is going up while that for $w$ is going down after cherry picking. This is consistent with our expectation since our cherry picking for $y$ is generating positive correlation between the treatment assignment, and the error term $\epsilon$ and $\epsilon$ is positively (negatively) correlated with $\eta(\gamma)$. As a result, the estimated treatment effect for $z$ is biased up while that for $w$ is biased down (see the last three columns in Table A3 for detailed results).

Our cherry picking approach can be extended for multiple outcome variables. In particular, we can run our algorithm separately for each of the outcome variables and obtain their corresponding scores. We can then pick observations based on, e.g., a weighted average of the scores, where the weights depend on the goal of cherry picking. ${ }^{6}$ For instance, suppose we are trying to establish a strong positive treatment effect for $y$ and reduce the treatment effect for $z$ to null. Since $\epsilon$ and $\eta$ are positively correlated, this is challenging. We need to balance the trade offs between biasing up the estimation for $y$ and biasing down the estimation for $z$. One simple way of doing this is to consider a weighted score that puts a positive weight on the score for $y$ and a negative weight on the score for $z{ }^{7}$

\footnotetext{
${ }^{6}$ In this case, the weights are tuning parameters. One can alternatively think of more complicated ways beyond simple weightings to model the trade offs.

${ }^{7}$ Table A4 shows results from a sample exercise where we cherry pick 500 observations with the highest
} 
While our algorithm selects a subset of data that is indistinguishable from a random sample, the complexities of real world data may render this type of p-hacking unrealistic in practice. In the next section we evaluate the algorithm's performance using publicly available replication data from a well-known randomized control trial.

\section{Cherry Picking for Positive Average Treatment Effects from Microfinance}

We use data from Banerjee et al. (2015) to explore the effects of the algorithm with an empirical, real world dataset. In their original paper, Banerjee et al. (2015) study the expansion of Spandana, a for-profit microcredit program in Hyderabad, India. They find modest increases in outcomes like business investment but null effects on a wide range of outcomes including consumption, health, education, and women's empowerment.

We apply our algorithm to select a subsample of the data in Banerjee et al. (2015) that will yield a positive and significant treatment effect on consumption, then test to see whether the selected subsample is different from the full sample. The setting and experimental design in Banerjee et al. (2015) differs from our simulation in two ways. First, the randomization occurs at the neighborhood, or area level. Second, the authors stratify the randomization by pre-experimental per capita consumption and debt levels. They identify 104 neighborhoods that Spandana is interested in expanding into then group those areas into pairs based on average per capita consumption and debt. In each pair, they randomly assign one area to treatment and the other to control.

Each observation in the endline data is a household. The authors form their sampling frame based on the households who they observe to be most likely to borrow, which are households who have resided in the area for at least three years and have at least one woman between 18 and 55 years of age. In practice the authors oversample Spandana borrowers, so we use the weights they provide in their replication data throughout our analysis.

Banerjee et al. (2015) use the following equation to estimate reduced-form impacts from Spandana's expansion:

$$
y_{i a}=\alpha+\beta \text { Treat }_{i a}+\mathbf{X}_{a}^{\prime} \gamma+\epsilon_{i a} .
$$

Here, $y_{i a}$ is an outcome of interest for household $i$ in area $a$, Treat $_{i a}$ is the treatment dummy variable which is equal to one for households located in a treated area, and $\mathbf{X}_{a}{ }_{a}$ is a vector of control variables, calculated as area-level baseline values: area population, total businesses, average per capita expenditure, fraction of household heads who are literate, and fraction of all adults who are literate. They cluster all standard errors at the area level.

weighted scores. 


\subsection{Selecting for a Positive ATE on Total Consumption}

First, we ask whether we can use our algorithm to select a subset of the replication data for Banerjee et al. (2015) that will yield a positive treatment effect on total monthly per capita consumption using equation (1). Table 6 in Banerjee et al. (2015) shows that total monthly per capita expenditures on consumption increase by 10.24 rupees, on average, in treated areas relative to control areas, with a standard error of 37.22 .

In Table 2, we successfully replicate the original result on total monthly per capita expenditure, presented in column (1). Columns (2) through (4) show ATEs on subsets of the data selected using our algorithm. In column (2), we select the $50 \%$ of the data with the highest scores assigned by our algorithm. Estimating equation (1) on this subset yields an ATE of 649.03, an effect which is 65 times larger than the true ATE estimated on the full sample. Columns (3) and (4) repeat this exercise with larger subsets of the original data: $75 \%$ and $95 \%$, respectively. Both subsets yield ATEs that are an order of magnitude larger than the true ATE and statistically significant at the $1 \%$ level.

Having confirmed that our algorithm can select a subset of real world data to yield large and statistically significant ATEs, we next ask how the selected subsets compare to the original dataset. First, we test to see whether there are significant differences in the covariates included in the regression for our selected subsample relative to the original dataset. We perform a two-sample Kolmogorov-Smirnov test to see if the distributions of each covariate differ between samples. All p-values are greater than 0.13 and most are close to one, indicating that we fail to reject that the distributions in the selected subsample are equal to those in the full dataset (see Table A5 for a complete list of p-values from Kolmogorov-Smirnov tests for all of the covariates included in equation 1 between the original dataset and our $50 \%, 75 \%$, and $95 \%$ selected subsamples).

Similar to our analysis for simulated data, we can also see that correlations are low between the score assigned by our algorithm and the covariates in the analysis. As expected, there is a statistically significant correlation of $12.8 \%$ between the score and total monthly consumption expenditures per capita, but none of the other covariates display a significant correlation with the score (see Table A6 for a complete list of correlations).

Having established that our algorithm produces similar results in a real world dataset to what we demonstrate in our simulation data, we now consider whether there are any natural deterrents to cherry picking for unscrupulous researchers. First, we consider whether the algorithm performs well if a pre-analysis plan were to outline a more complicated sampling strategy. Next, we test the robustness of the results in our selected subset. Finally, we ask whether the algorithm forces the researcher to pick one outcome at the expense of others. 
Table 2: Selecting for Positive ATEs on Total Consumption

\begin{tabular}{|c|c|c|c|c|}
\hline & \multicolumn{4}{|c|}{ Dependent variable: } \\
\hline & (Full Sample) & $\begin{array}{l}\text { Total Monthly Per } \\
\text { (50\% Subsample) }\end{array}$ & $\begin{array}{l}\text { Capita Consumption } \\
\qquad \text { (75\% Subsample) }\end{array}$ & (95\% Subsample) \\
\hline & $(1)$ & $(2)$ & $(3)$ & $(4)$ \\
\hline treatment & $\begin{array}{c}10.243 \\
(37.217)\end{array}$ & $\begin{array}{c}668.262^{* * *} \\
(50.771)\end{array}$ & $\begin{array}{c}364.698^{* * *} \\
(36.274)\end{array}$ & $\begin{array}{c}149.745^{* * *} \\
(33.256)\end{array}$ \\
\hline area_pop_base & $\begin{array}{l}-0.053 \\
(0.127)\end{array}$ & $\begin{array}{c}-0.173 \\
(0.139)\end{array}$ & $\begin{array}{l}-0.083 \\
(0.114)\end{array}$ & $\begin{array}{l}-0.059 \\
(0.113)\end{array}$ \\
\hline area_debt_total_base & $\begin{array}{c}-0.001 \\
(0.0004)\end{array}$ & $\begin{array}{c}0.001 \\
(0.0005)\end{array}$ & $\begin{array}{c}0.0003 \\
(0.0004)\end{array}$ & $\begin{array}{l}-0.0003 \\
(0.0004)\end{array}$ \\
\hline area_business_total_base & $\begin{array}{c}-11.815^{* * *} \\
(3.903)\end{array}$ & $\begin{array}{c}-14.155^{* *} \\
(5.738)\end{array}$ & $\begin{array}{c}-13.396^{* * *} \\
(3.964)\end{array}$ & $\begin{array}{c}-13.171^{* * *} \\
(3.693)\end{array}$ \\
\hline area_exp_pc_mean_base & $\begin{array}{c}0.685^{* * *} \\
(0.088)\end{array}$ & $\begin{array}{c}0.809^{* * *} \\
(0.154)\end{array}$ & $\begin{array}{c}0.644^{* * *} \\
(0.092)\end{array}$ & $\begin{array}{c}0.658^{* * *} \\
(0.083)\end{array}$ \\
\hline area_literate_head_base & $\begin{array}{c}-335.842^{*} \\
(195.326)\end{array}$ & $\begin{array}{c}-500.869^{* *} \\
(223.643)\end{array}$ & $\begin{array}{c}-396.138^{* *} \\
(182.607)\end{array}$ & $\begin{array}{l}-328.255^{*} \\
(179.486)\end{array}$ \\
\hline area_literate_base & $\begin{array}{c}1,016.152^{* * *} \\
(330.395)\end{array}$ & $\begin{array}{c}1,018.644^{* *} \\
(482.688)\end{array}$ & $\begin{array}{c}890.224^{* * *} \\
(326.709)\end{array}$ & $\begin{array}{c}856.960^{* * *} \\
(306.299)\end{array}$ \\
\hline Constant & $\begin{array}{l}392.898^{* *} \\
(172.992)\end{array}$ & $\begin{array}{c}81.757 \\
(245.679) \\
\end{array}$ & $\begin{array}{l}334.874^{* *} \\
(165.847)\end{array}$ & $\begin{array}{l}405.501^{* * *} \\
(154.899)\end{array}$ \\
\hline Observations & 6,827 & 3,415 & 5,122 & 6,483 \\
\hline F Statistic & $14.147^{* * *}(\mathrm{df}=7 ; 6819)$ & $49.234^{* * *}(\mathrm{df}=7 ; 3407)$ & $35.439^{* * *}(\mathrm{df}=7 ; 5114)$ & $21.757^{* * *}(\mathrm{df}=7 ; 6475)$ \\
\hline
\end{tabular}

Note: $\quad{ }^{*} \mathrm{p}<0.1 ;{ }^{* *} \mathrm{p}<0.05 ;{ }^{* * *} \mathrm{p}<0.01$

Average treatment effects on total consumption expenditures on the full datset (replicating Banerjee et al (2015)) and a range of subsets selected using our algorithm. We cluster all standard errors at the area level. 


\subsection{Natural Deterrents to Cherry Picking}

The exercise above shows that a researcher could select observations to generate large treatment effects in a manner that is undetectable ex post. However, many experimental designs involve more complex sampling and randomization strategies, which are typically detailed in pre-analysis plans. We evaluate the performance of our algorithm when we restrict the selected subsample to follow a stratified sampling strategy.

The full sample in Banerjee et al. (2015) is drawn in roughly equal proportions from 104 different neighborhoods. We calculate the precise proportion of the sample drawn from each neighborhood in the full sample, then calculate the corresponding number of observations that would need to come from each neighborhood to ensure that the overall distribution of respondents across neighborhoods is identical in selected subsamples as they are in the full sample. Again, we consider subsamples of $50 \%, 75 \%$, and $95 \%$. The algorithm remains the same, but we include one extra step in selecting the subsample after running the algorithm in which we ensure that the correct proportion of the selected sample comes from each neighborhood. If restricting our selection to follow the pre-specified stratification leads us to select a subset that does not yield large and statistically significant ATEs, it indicates that complex sampling acts as a natural deterrent to cherry picking.

We find that mimicking a more complex sampling strategy does lead to lower ATEs in our selected subsamples; however, the reduction in ATEs is small relative to the massive increase generated by selecting based on the scores from our algorithm. For $50 \%, 75 \%$, and 95\% subsamples, the estimated ATEs remain the same order of magnitude as the ATEs we estimate without restricting subsamples to follow the more complex sampling strategy (see Table A7 for a full comparison of manipulated ATEs for the unrestricted subsamples versus those that we restrict to follow the same distribution across neighborhoods as the original sample). It does not appear that complex sampling strategies alone provide a strong deterrent to dataset manipulation.

An obvious limitation to our algorithm is that a researcher can only use it to select data that will yield a significant ATE for the specification used in the algorithm. We consider whether the selected subsamples yield results that are robust to a range of alternative specifications that a researcher might reasonably be expected to present. For our robustness results, we present results from the full sample compared to the $95 \%$ subsample, which we view as the most realistic case of researcher fraud.

First, we simply omit the weights provided for all regressions. The results in the original paper are robust to omitting weights: coefficients remain stable and levels of significance do not change. We find that the same is true of our $95 \%$ subsample. Omitting weights barely changes point estimates or standard errors (see Table A8). 
Next, we follow Brodeur, Cook, and Heyes (2020) to test robustness to every possible combination of control variables. As a first pass, we only consider the six control variables that Banerjee et al. (2015) include in their analysis. All cominations of these six control variables yield ATEs of a similar magnitude (145-175) and with similar t-statistics (around 4). This suggests that the ATEs we estimate in our selected subsample are robust to a wide range of alternative, linear specifications (see figure A1 for the full output from the Stata command provided by Brodeur, Cook, and Heyes (2020)). Alternatively, we repeat the same exercise but include an additional six household-level baseline covariates as possible control variables. The logic behind this decision is that Brodeur, Cook, and Heyes (2020) recommend testing all combinations of control variables in the data. If an unscrupulous researcher provided the baseline covariates, it is reasonable to assume that a peer reviewer may ask to see the robustness check proposed by Brodeur, Cook, and Heyes (2020) for all control variables. Using twelve control variables still indicates that effects are large and statistically significant, as all t-statistics are over 2 and all effect sizes fall between 120 and 175 (see figure A2 for full results). Such a robustness check does not provide a natural deterrent to dataset manipulation.

While Brodeur, Cook, and Heyes (2020) tests all combinations of control variables, our results may be less robust to alternative functional forms. We apply an inverse hyperbolic sine transformation to total monthly per capita consumption expenditures and find that our $95 \%$ selected subset still yields an ATE that is large and statistically significant (see Table A9).

Finally, we consider the measure of robustness proposed in Broderick, Giordano, and Meager (2021), which calculates the amount of data that would need to be removed to change the significance level of the result, the sign of the result, or both. With our $95 \%$ selected subset, dropping only 69 observations (1.1\% of the data) changes the significance of our result. As expected, our $75 \%$ sample is substantially more robust, requiring that we drop 453 observations ( $9 \%$ of the data) to change the level of significance. As discussed in the context of our simulated data, it is not surprising that our selected subsets do not stand up to the robustness measure in Broderick, Giordano, and Meager (2021), as their measure relies on similar logic to our algorithm but does not restrict the datasets that result from dropping influential results to be "undetectable" as we do.

From a practical perspective, it is unclear whether the robustness check proposed by Broderick, Giordano, and Meager (2021) is sufficient to deter data manipulation. They highlight a number of well-regarded studies that display similar robustness properties to our 95\% selected subsample. We do not believe that any of those studies engaged in the type of dataset manipulation we are discussing, suggesting that failing to pass the Broderick, 
Giordano, and Meager (2021) robustness check is not a reliable indicator of manipulation.

In practice, most RCTs test multiple outcomes. Selecting data to yield a positive ATE on one may yield insignificant effects on other outcomes of interest, potentially mitigating concerns about dataset manipulation. We explore this question by comparing estimated ATEs in the original sample from Banerjee et al. (2015) to the subsamples that we selected to generate a positive ATE on total monthly per capita consumption. Throughout, we use a large, $95 \%$ subsample. We do not change the algorithm to weight multiple outcomes of interest. We focus on two families of outcomes: measures of business growth and success and measures of consumption (Tables 3 and 6 in Banerjee et al. (2015), respectively).

Table 3 shows our results on multiple outcomes. Replicating the results of the original paper, we find that only business investment and the index of all business measures combined are statistically significant using the full sample. Both are only significant at the $10 \%$ level. Using our 95\% sample selected on monthly per capita consumption expenditures, we estimate ATEs that are larger on all measures of business outcomes. Our ATEs on assets, investment, starting a new business, and the overall index of business measures are all significant at the $5 \%$ level, and our ATE on profits is significant at the 10\% level. The increase in the size and significance of the ATEs in our selected subsample generally occur in the outcomes with the highest correlations with consumption expenditure. However, the correlation between profits and consumption expenditure is only 0.05 and the ATE in our selected subsample is over $150 \%$ the ATE in the full sample. Overall, using our selected $95 \%$ subsample would make it appear as though expanding microcredit leads to significant improvements in business outcomes.

By contrast, when we compare results between the original sample and our selected subsample for the range of consumption outcomes covered in Table 6 of Banerjee et al. (2015) we see that a number of outcomes with null effects in the full sample exhibit large, positive effects in our selected subsample. Expenditures on durables, nondurables, and health are all larger in our 95\% selected subsample, and statistically significant at the $1 \%$ level. Given that these expenditure categories likely make up a large proportion of total per capita consumption expenditures, the outcome that we used to select our subsample, our results are not surprising. Critically, a number of outcomes with significant, negative ATEs in the original sample have ATEs that are closer to zero and are not statistically significant in our 95\% selected subsample. Banerjee et al. (2015) find negative impacts on spending on temptation goods as well as festivals and celebrations. Since our subsample is selected on total consumption expenditures, it does not preserve these negative treatment effects (see Table A10 for full results on consumption outcomes).

As discussed previously, a researcher could trade off between these outcomes in an effort 
Table 3: ATEs on Business Outcomes After Selecting on Consumption

\begin{tabular}{|c|c|c|c|c|c|c|c|c|}
\hline & \multicolumn{8}{|c|}{ Dependent variable: } \\
\hline & \multicolumn{2}{|c|}{ Assets } & \multicolumn{2}{|c|}{ Investment } & \multicolumn{2}{|c|}{ Expenses } & \multicolumn{2}{|c|}{ Profits } \\
\hline & Full & $95 \%$ & Full & $95 \%$ & Full & $95 \%$ & Full & $95 \%$ \\
\hline & $(1)$ & $(2)$ & $(3)$ & $(4)$ & $(5)$ & $(6)$ & $(7)$ & $(8)$ \\
\hline treatment & $\begin{array}{c}597.510 \\
(383.518)\end{array}$ & $\begin{array}{l}792.532^{* *} \\
(396.559)\end{array}$ & $\begin{array}{l}390.853^{*} \\
(212.695)\end{array}$ & $\begin{array}{l}543.752^{* *} \\
(211.858)\end{array}$ & $\begin{array}{c}254.664 \\
(1,056.330)\end{array}$ & $\begin{array}{c}1,098.800 \\
(1,043.403)\end{array}$ & $\begin{array}{c}354.338 \\
(313.531)\end{array}$ & $\begin{array}{l}563.665^{*} \\
(300.361)\end{array}$ \\
\hline \multirow[t]{4}{*}{ Observations } & $\begin{array}{c}0.14 \\
6,800\end{array}$ & 6,459 & $\begin{array}{c}0.15 \\
6,800\end{array}$ & 6,459 & $\begin{array}{c}0.11 \\
6,685\end{array}$ & 6,355 & $\begin{array}{c}0.05 \\
6,239\end{array}$ & 5,930 \\
\hline & \multicolumn{2}{|c|}{ Any Business } & \multicolumn{2}{|c|}{ No. Businesses } & \multicolumn{2}{|c|}{ New Business } & \multicolumn{2}{|c|}{ Index } \\
\hline & Full & $95 \%$ & Full & $9 \%$ & Full & $95 \%$ & Full & $95 \%$ \\
\hline & $(1)$ & $(2)$ & $(3)$ & $(4)$ & $(5)$ & $(6)$ & $(7)$ & $(8)$ \\
\hline treatment & $\begin{array}{c}0.008 \\
(0.021)\end{array}$ & $\begin{array}{c}0.013 \\
(0.021)\end{array}$ & $\begin{array}{c}0.018 \\
(0.038)\end{array}$ & $\begin{array}{c}0.030 \\
(0.038)\end{array}$ & $\begin{array}{c}0.009 \\
(0.006)\end{array}$ & $\begin{array}{l}0.013^{* *} \\
(0.006)\end{array}$ & $\begin{array}{c}0.036^{*} \\
(0.019) \\
\end{array}$ & $\begin{array}{c}0.054^{* * *} \\
(0.018)\end{array}$ \\
\hline Corr with Consumption & 0.07 & & 0.08 & & 0.04 & & 0.18 & \\
\hline Observations & 6,810 & 6,469 & 6,810 & 6,469 & 6,757 & 6,424 & 6,810 & 6,469 \\
\hline
\end{tabular}

ATEs of outcomes other than consumption expenditures. Columns (1), (3), (5), and (7) replicate the results reported in Banerjee et al (2015) Table 3. All regression include area-level baseline controls for population, debt, businesses, expenditures, and literacy that Banerjee et al (2015) use throughout their analysis, but we omit coefficients on these controls for brevity. We cluster standard errors at the area level. 
to preserve both the positive treatment effects on items like durables, nondurables, and health and the negative ATEs on festivals and temptation goods. With the large number of outcomes examined in only two of many tables in Banerjee et al. (2015), this may not be feasible in practice. Given that we find positive and significant ATEs on a range of outcomes exhibiting relatively small, positive correlations with the variable we use to select our subset, we do not view multiple outcomes as a strong deterrent to dataset manipulation.

\section{Discussion and Conclusion}

We have shown that an unscrupulous researcher can use our straightforward algorithm to select a large subset of data that yields ATEs that are an order of magnitude larger than the true effects in the full dataset. The selection is undetectable ex post in the sense that covariates remain balanced between treatment and control and even a reviewer with access to an independent sample of data from the population of interest could not reject that the covariates are distributed identically. The algorithm continues to perform well under more complex sampling strategies, the results in our selected subsample stand up to a range of robustness checks, and we estimate multiple large and statistically significant ATEs on outcomes other than the one we use to select our subsample. Taken together, our results indicate that there are not strong natural deterrents to dataset manipulation.

This type of cherry picking could easily go undetected under the current pre-analysis plan regime. For instance, an unscrupulous researcher could claim that they were only able to collect $95 \%$ of the data they had originally intended to collect due to unexpected budget constraints, or they could plan from the outset to collect $5 \%$ more data than stated in their pre-analysis plan. If the researcher only provides the selected subset upon publication, all of their estimated results would replicate.

We propose a straightforward innovation on current data transparency practices that can prevent researchers from manipulating datasets at minimal cost to researchers and reviewers: a secure metadata clearinghouse. The idea behind the clearinghouse is that the type of manipulation we describe can be prevented with minimal knowledge about the original dataset, and without the need to share any actual data. If scholars can credibly report the number of non-missing observations for each variable, any deviations between the original dataset and the dataset used in the final publication would be obvious and researchers would need to clearly document and justify any data exclusions.

Credibly reporting the number of non-missing observations does pose a challenge. Here, we rely on the institutional structures involved in running field experiments. Namely, researchers are rarely the first to interact with the data used in RCTs. Survey firms and 
enumerators are typically the first to record and upload primary data. Where experiments rely on administrative data, the data often originate from governments or private companies. These third parties may be able to directly provide the metadata we recommend or to verify the accuracy of metadata submitted by a researcher. Similar principles apply to lab experiments, as principal investigators are rarely soley responsible for primary data collection.

While our proposed solution theoretically protects against dataset manipulation, we recognize the potential for researcher abuse of power. Principal investigators often wield considerable financial and reputational power in scenarios where they hire local survey teams, work with local partners, or hire research assistants who may feel pressured to under-report metadata if asked. A fully functional metadata clearinghouse will need clear protocols for whistle-blowers to come forward anonymously and strategies to educate third party users independently of information being provided by the researchers they are working for. 


\section{Appendix}

Table A1: Summary of All Simulated Data and Covariates Balance

\begin{tabular}{|c|c|c|c|c|}
\hline Statistic & Mean & St. Dev. & Min & Max \\
\hline $\mathrm{y}$ & 0.114 & 0.970 & -3.118 & 3.125 \\
\hline $\mathrm{z}$ & -0.244 & 0.865 & -3.162 & 2.417 \\
\hline $\mathrm{W}$ & 0.171 & 0.764 & -2.189 & 2.500 \\
\hline $\mathrm{x} 1$ & 0.022 & 0.984 & -3.355 & 3.622 \\
\hline $\mathrm{x} 2$ & 0.013 & 0.970 & -2.700 & 3.160 \\
\hline \multirow[t]{2}{*}{ treat } & 0.292 & 0.455 & 0 & 1 \\
\hline & Control & Treatment & Diff/SD & \\
\hline $\mathrm{x} 1$ & 0.022 & 0.021 & 0.001 & \\
\hline $\mathrm{x} 2$ & 0.013 & 0.011 & 0.003 & \\
\hline
\end{tabular}

Table A2: Summary of 75\% Cherry Picked Data and Covariates Balance

\begin{tabular}{|c|c|c|c|c|}
\hline Statistic & Mean & St. Dev. & Min & Max \\
\hline $\mathrm{y}$ & 0.111 & 0.962 & -3.118 & 3.119 \\
\hline $\mathrm{x} 1$ & 0.033 & 0.970 & -3.355 & 3.622 \\
\hline $\mathrm{x} 2$ & 0.020 & 0.966 & -2.700 & 3.160 \\
\hline \multirow[t]{2}{*}{ treat } & 0.297 & 0.457 & 0 & 1 \\
\hline & Control & Treatment & \multicolumn{2}{|c|}{ Diff/SD } \\
\hline $\mathrm{x} 1$ & 0.027 & 0.047 & \multicolumn{2}{|c|}{-0.021} \\
\hline$x 2$ & 0.020 & 0.021 & \multicolumn{2}{|c|}{-0.001} \\
\hline
\end{tabular}


Table A3: Linear Regressions of $y, z, w$ Before/After Cherry Picking

\begin{tabular}{|c|c|c|c|c|c|c|}
\hline & \multicolumn{6}{|c|}{ Dependent variable: } \\
\hline & $\begin{array}{c}\mathrm{y} \\
(1)\end{array}$ & $\begin{array}{c}\mathrm{Z} \\
(2) \\
\end{array}$ & $\begin{array}{l}\mathrm{w} \\
(3)\end{array}$ & $\begin{array}{c}\mathrm{y} \\
(4)\end{array}$ & $\begin{array}{c}\mathrm{Z} \\
(5)\end{array}$ & $\begin{array}{l}\mathrm{w} \\
(6)\end{array}$ \\
\hline treat & $\begin{array}{c}0.074^{*} \\
(0.043)\end{array}$ & $\begin{array}{c}0.181^{* * *} \\
(0.035)\end{array}$ & $\begin{array}{c}-0.143^{* * *} \\
(0.023)\end{array}$ & $\begin{array}{c}0.254^{* * *} \\
(0.049)\end{array}$ & $\begin{array}{c}0.298^{* * *} \\
(0.040)\end{array}$ & $\begin{array}{c}-0.207^{* * *} \\
(0.027)\end{array}$ \\
\hline $\mathrm{x} 1$ & $\begin{array}{c}-0.127^{* * *} \\
(0.028)\end{array}$ & $\begin{array}{c}-0.645^{* * *} \\
(0.022)\end{array}$ & $\begin{array}{c}0.752^{* * *} \\
(0.015)\end{array}$ & $\begin{array}{c}-0.111^{* * *} \\
(0.031)\end{array}$ & $\begin{array}{c}-0.631^{* * *} \\
(0.026)\end{array}$ & $\begin{array}{c}0.747^{* * *} \\
(0.017)\end{array}$ \\
\hline $\mathrm{x} 2$ & $\begin{array}{c}-0.318^{* * *} \\
(0.028)\end{array}$ & $\begin{array}{c}0.262^{* * *} \\
(0.022)\end{array}$ & $\begin{array}{c}-0.793^{* * *} \\
(0.015)\end{array}$ & $\begin{array}{c}-0.330^{* * *} \\
(0.031)\end{array}$ & $\begin{array}{c}0.243^{* * *} \\
(0.026)\end{array}$ & $\begin{array}{c}-0.792^{* * *} \\
(0.017)\end{array}$ \\
\hline Constant & $\begin{array}{c}0.099^{* * *} \\
(0.023)\end{array}$ & $\begin{array}{c}-0.286^{* * *} \\
(0.019)\end{array}$ & $\begin{array}{c}0.207^{* * *} \\
(0.013)\end{array}$ & $\begin{array}{c}0.046^{*} \\
(0.027)\end{array}$ & $\begin{array}{c}-0.308^{* * *} \\
(0.022)\end{array}$ & $\begin{array}{c}0.225^{* * *} \\
(0.015)\end{array}$ \\
\hline Observations & 2,000 & 2,000 & 2,000 & 1,500 & 1,500 & 1,500 \\
\hline $\mathrm{R}^{2}$ & 0.175 & 0.337 & 0.612 & 0.186 & 0.336 & 0.620 \\
\hline
\end{tabular}

The first three columns are from linear regressions using all 2000 observations. The last three columns are from linear regressions using cherry picked 1500 observations. 
Table A4: Linear Regressions of $y, z$ Before/After Weighted Cherry Picking

\begin{tabular}{|c|c|c|c|c|}
\hline & \multicolumn{4}{|c|}{ Dependent variable: } \\
\hline & $\begin{array}{c}\mathrm{y} \\
(1)\end{array}$ & $\begin{array}{c}\mathrm{Z} \\
(2) \\
\end{array}$ & $\begin{array}{c}\mathrm{y} \\
(3)\end{array}$ & $\begin{array}{c}\mathrm{Z} \\
(4)\end{array}$ \\
\hline treat & $\begin{array}{c}0.074^{*} \\
(0.043)\end{array}$ & $\begin{array}{c}0.181^{* * *} \\
(0.035)\end{array}$ & $\begin{array}{c}0.365^{* * *} \\
(0.080)\end{array}$ & $\begin{array}{l}0.137^{* *} \\
(0.065)\end{array}$ \\
\hline $\mathrm{x} 1$ & $\begin{array}{c}-0.127^{* * *} \\
(0.028)\end{array}$ & $\begin{array}{c}-0.645^{* * *} \\
(0.022)\end{array}$ & $\begin{array}{c}-0.144^{* * *} \\
(0.054)\end{array}$ & $\begin{array}{c}-0.661^{* * *} \\
(0.044)\end{array}$ \\
\hline $\mathrm{x} 2$ & $\begin{array}{c}-0.318^{* * *} \\
(0.028)\end{array}$ & $\begin{array}{c}0.262^{* * *} \\
(0.022)\end{array}$ & $\begin{array}{c}-0.272^{* * *} \\
(0.053)\end{array}$ & $\begin{array}{c}0.261^{* * *} \\
(0.043)\end{array}$ \\
\hline Constant & $\begin{array}{c}0.099^{* * *} \\
(0.023)\end{array}$ & $\begin{array}{c}-0.286^{* * *} \\
(0.019)\end{array}$ & $\begin{array}{l}-0.022 \\
(0.047)\end{array}$ & $\begin{array}{c}-0.269^{* * *} \\
(0.038)\end{array}$ \\
\hline Observations & 2,000 & 2,000 & 500 & 500 \\
\hline $\mathrm{R}^{2}$ & 0.175 & 0.337 & 0.187 & 0.342 \\
\hline
\end{tabular}

The first two columns are from linear regressions using all 2000 observations. The last two columns are from linear regressions using cherry picked data, which is picked based on the weighted scores and contains 500 observations. The weighted scores are calculated by $1.01 \cdot \operatorname{score}(y)-1.13 \cdot \operatorname{score}(z)$. 
Table A5: Kolmogorov-Smirnov p-values Comparing Selected Subsets to Full Dataset

\begin{tabular}{cccc}
\hline \hline & K-S p-value 50\% & K-S p-value 75\% & K-S p-value 95\% \\
\hline total_exp_mo_pc_1 & 0.997 & 0.574 & 0.713 \\
treatment & 0.135 & 0.901 & 1 \\
area_pop_base & 0.994 & 1.000 & 1 \\
area_debt_total_base & 0.799 & 1.000 & 1 \\
area_business_total_base & 0.904 & 1.000 & 1 \\
area_exp_pc_mean_base & 0.976 & 1 & 1 \\
area_literate_head_base & 0.769 & 1.000 & 1 \\
area_literate_base & 0.820 & 1.000 & 1 \\
\hline
\end{tabular}

Note: p-values from Kolmogorov-Smirnov tests comparing the distribution of variables between the full dataset and our selected subsets. The null hypothesis is that the distributions in the two datasets are equal, so high p-values indicate that we fail to reject equal distributions.

Table A6: Correlation Between Algorithm-Assigned Score and Covariates

\begin{tabular}{cc}
\hline \hline & Score \\
\hline total_exp_mo_pc_1 & 0.128 \\
treatment & -0.009 \\
area_pop_base & -0.015 \\
area_debt_total_base & 0.012 \\
area_business_total_base & -0.001 \\
area_exp_pc_mean_base & 0.018 \\
area_literate_head_base & 0.014 \\
area_literate_base & -0.002 \\
\hline
\end{tabular}

Note: Only the correlation between total per capita monthly consumption expenditures and the score is statistically significant at the $5 \%$ level. 
Table A7: Selecting for Positive ATEs on Consumption with More Restrictive Sampling

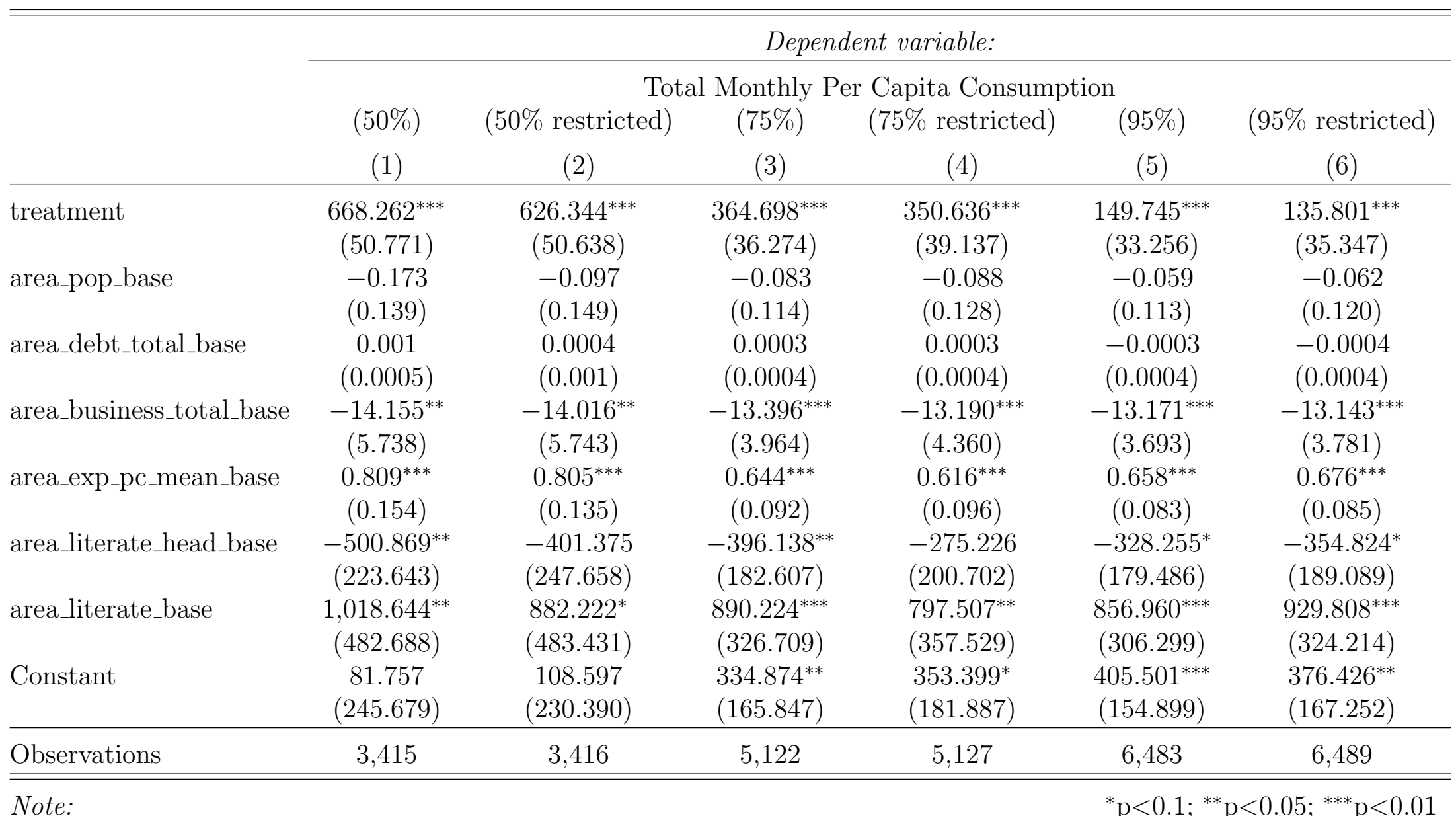

Average treatment effects on subsets of data selected using our algorithm. In restricted subsamples, we impose the additional requirement that the proportion of respondents from each neighborhood is the same as in the full dataset. This mimics what an unscrupulous researcher might do when attempting to manipulate data in a way that would not be detectable after they registered a more complex sampling strategy such as stratification by neighborhood. We cluster all standard errors at the area level. 
Table A8: Robustness to Different Weights

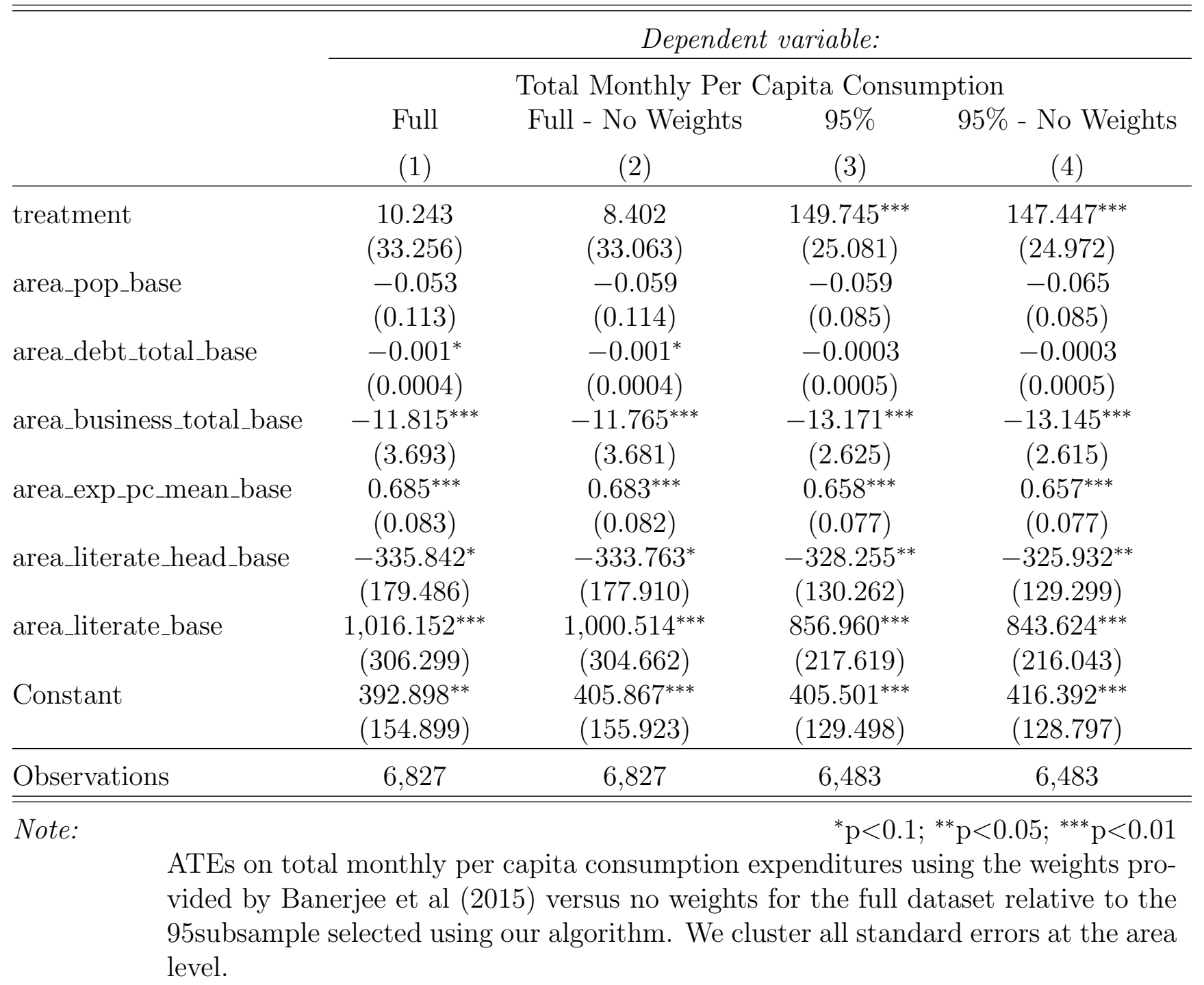


Figure A1: Robustness to All Combinations of Covariates Used in Banerjee et al (2015)
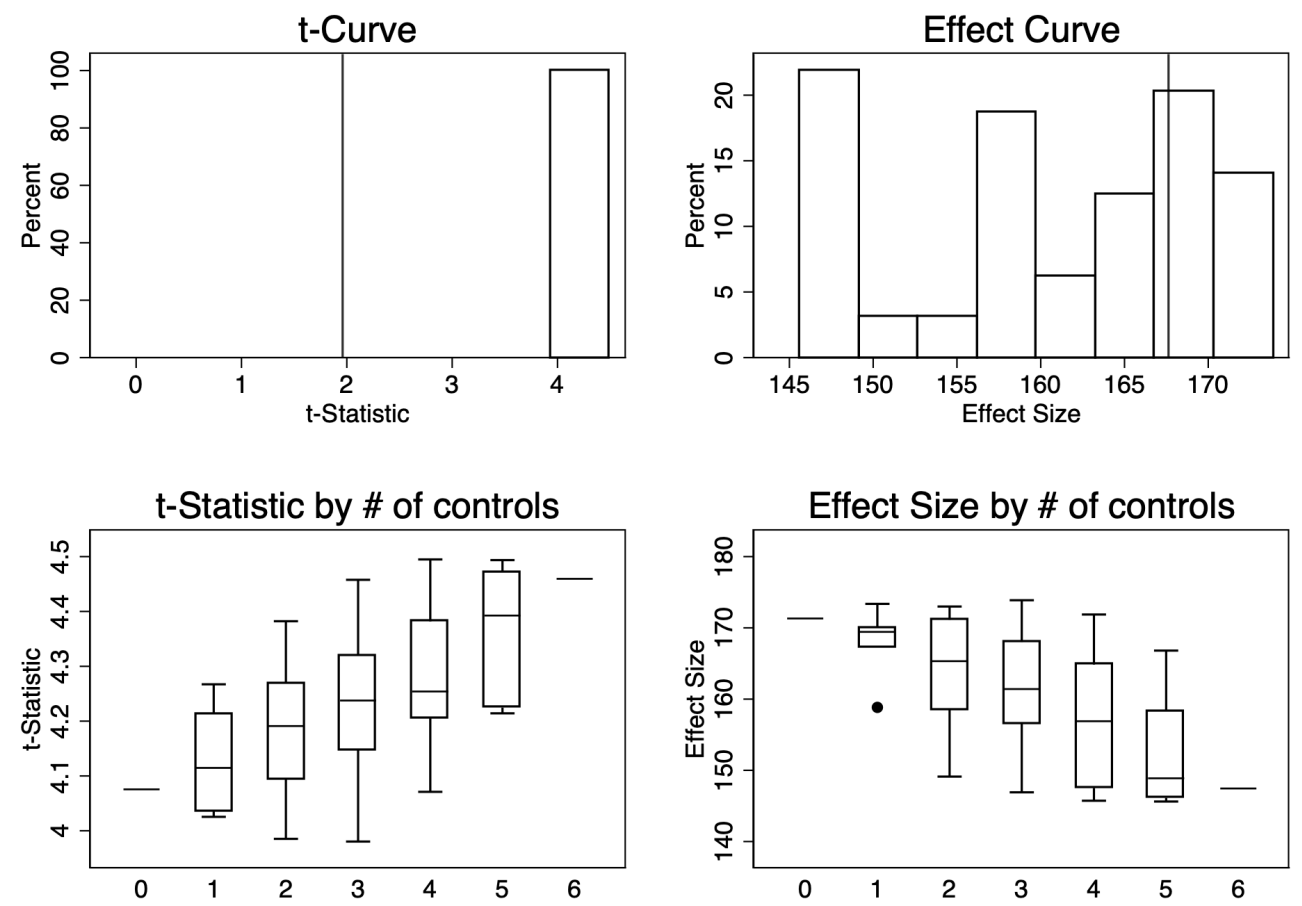

Note: t-curve shows the distribution of t-statistics across all specifications. Effect curve shows the distribution of effect sizes across all specifications. The bottom two panels show the distribution of t-statistics (left) and effect sizes (right) for different numbers of control variables. In this analysis, we only consider the six control variables that Banerjee et al (2015) include in their analysis. 
Figure A2: Robustness to All Combinations of Expanded Covariates
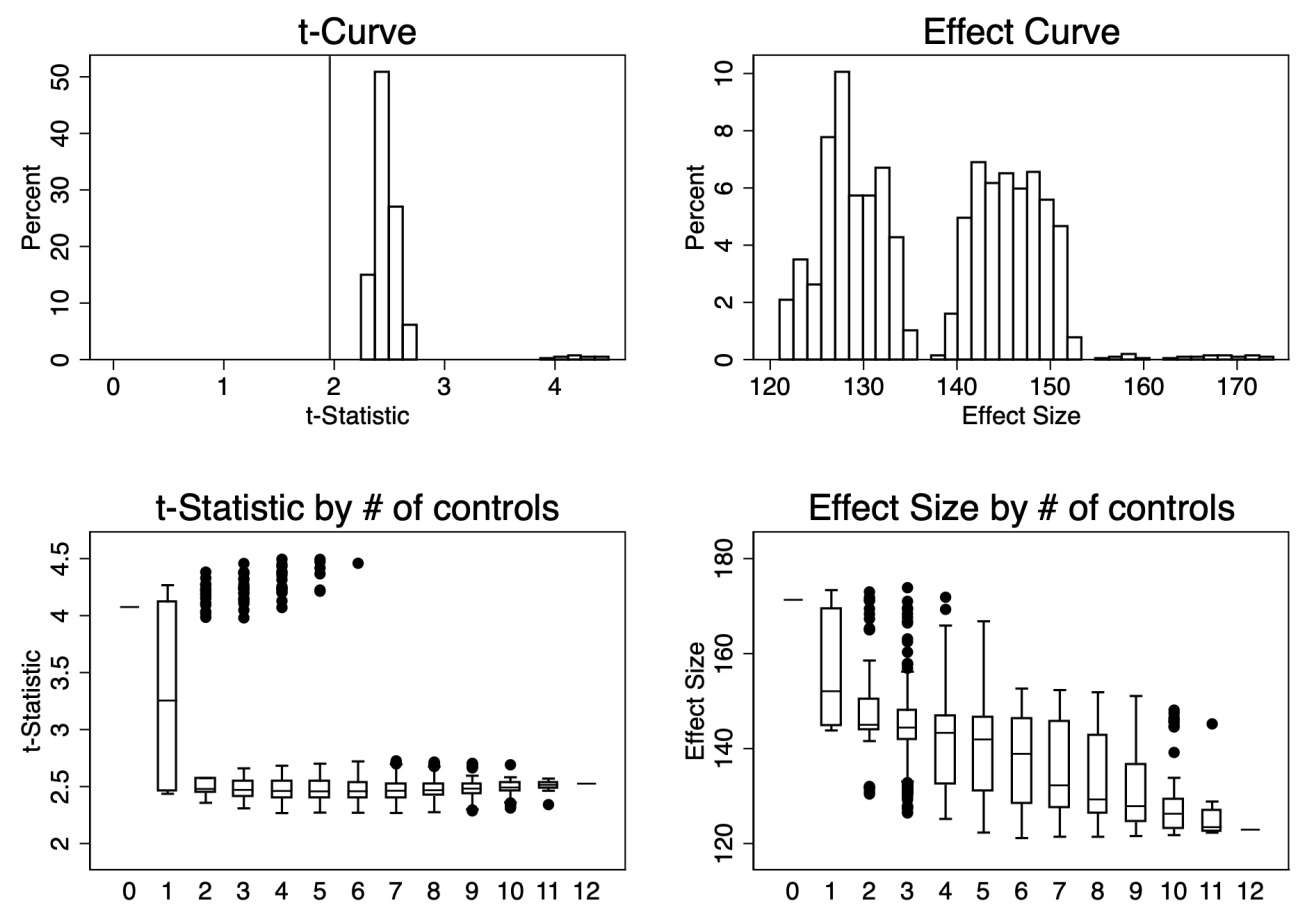

Note: t-curve shows the distribution of t-statistics across all specifications. Effect curve shows the distribution of effect sizes across all specifications. The bottom two panels show the distribution of t-statistics (left) and effect sizes (right) for different numbers of control variables. In this analysis, we consider the six control variables that Banerjee et al (2015) include in their analysis as well as an additional six household-level baseline controls that, for instance, a peer reviewer might expect to be included in this type of robustness check. 
Table A9: ATEs on Inverse Hyperbolic Since Transformation of Consumption (95\% Subsample)

\begin{tabular}{lc}
\hline \hline & Dependent variable: \\
\cline { 2 - 2 } & IHS(Consumption) \\
\hline treatment & $0.052^{* *}$ \\
& $(0.022)$ \\
area_pop_base & $(0.00003$ \\
& $(0.0001)$ \\
area_debt_total_base & $-0.00000^{*}$ \\
& $(0.00000)$ \\
area_business_total_base & $-0.010^{* * *}$ \\
& $(0.002)$ \\
area_exp_pc_mean_base & $0.0004^{* * *}$ \\
& $(0.0001)$ \\
area_literate_head_base & $-0.218^{*}$ \\
area_literate_base & $(0.127)$ \\
Constant & $0.655^{* * *}$ \\
& $(0.209)$ \\
& $6.529^{* * *}$ \\
Observations & $(0.114)$ \\
\hline \hline Note: & 6,483 \\
Average treatment effects on the invesrse hyperbolic since of total per capita monthly & ${ }^{*} \mathrm{p}<0.1 ;{ }^{* *} \mathrm{p}<0.05 ;{ }^{* * *} \mathrm{p}<0.01$ \\
consumption expenditures for the 95standard errors at the area level. &
\end{tabular}


Table A10: ATEs on Consumption Outcomes After Selecting on Total Consumption

\begin{tabular}{|c|c|c|c|c|c|c|c|c|}
\hline & \multicolumn{8}{|c|}{ Dependent variable: } \\
\hline & \multicolumn{2}{|c|}{ Durables } & \multicolumn{2}{|c|}{ Nondurables } & \multicolumn{2}{|c|}{ Food } & \multicolumn{2}{|c|}{ Health } \\
\hline & Full & $95 \%$ & Full & $95 \%$ & Full & $95 \%$ & Full & $95 \%$ \\
\hline & $(1)$ & $(2)$ & $(3)$ & $(4)$ & $(5)$ & $(6)$ & $(7)$ & $(8)$ \\
\hline treatment & $\begin{array}{c}19.734^{*} \\
(11.353)\end{array}$ & $\begin{array}{c}49.504^{* * *} \\
(10.925)\end{array}$ & $\begin{array}{c}-6.495 \\
(31.808)\end{array}$ & $\begin{array}{c}104.897^{* * *} \\
(28.312)\end{array}$ & $\begin{array}{l}-12.110 \\
(12.058)\end{array}$ & $\begin{array}{c}11.723 \\
(11.404)\end{array}$ & $\begin{array}{c}-3.700 \\
(11.514)\end{array}$ & $\begin{array}{c}26.910^{* * *} \\
(9.787)\end{array}$ \\
\hline \multirow[t]{4}{*}{ Observations } & $\begin{array}{c}0.46 \\
6,781 \\
\end{array}$ & 6,443 & $\begin{array}{c}0.77 \\
6,781 \\
\end{array}$ & 6,443 & $\begin{array}{c}0.58 \\
6,827 \\
\end{array}$ & 6,485 & $\begin{array}{c}0.46 \\
6,827 \\
\end{array}$ & 6,485 \\
\hline & \multicolumn{2}{|c|}{ Education } & \multicolumn{2}{|c|}{ Temptation } & \multicolumn{2}{|c|}{ Festivals } & \multicolumn{2}{|c|}{ Index } \\
\hline & Full & $95 \%$ & Full & $95 \%$ & Full & $95 \%$ & Full & $95 \%$ \\
\hline & $(1)$ & $(2)$ & $(3)$ & $(4)$ & $(5)$ & $(6)$ & $(7)$ & $(8)$ \\
\hline treatment & $\begin{array}{l}-2.061 \\
(9.865)\end{array}$ & $\begin{array}{c}9.075 \\
(9.283) \\
\end{array}$ & $\begin{array}{c}-8.785^{*} \\
(4.915)\end{array}$ & $\begin{array}{l}-3.793 \\
(4.894)\end{array}$ & $\begin{array}{c}-14.158^{*} \\
(8.094)\end{array}$ & $\begin{array}{l}-8.772 \\
(8.036)\end{array}$ & $\begin{array}{l}-0.051 \\
(0.057)\end{array}$ & $\begin{array}{l}-0.006 \\
(0.056)\end{array}$ \\
\hline Corr with Consumption & 0.42 & & 0.32 & & 0.27 & & 0.32 & \\
\hline Observations & 5,415 & 5,161 & 6,827 & 6,485 & 6,827 & 6,485 & 6,841 & 6,499 \\
\hline
\end{tabular}

ATEs of consumption outcomes other than total consumption expenditures. Columns (1), (3), (5), and (7) replicate the results reported in Banerjee et al (2015) Table 6. All regression include area-level baseline controls for population, debt, businesses, expenditures, and literacy that Banerjee et al (2015) use throughout their analysis, but we omit coefficients on these controls for brevity. We cluster standard errors at the area level. 


\section{References}

Angelucci, Manuela, Dean Karlan, and Jonathan Zinman (2015). "Microcredit Impacts: Evidence from a Randomized Microcredit Program Placement Experiment by Compartamos Banco". American Economic Journal: Applied Economics 7, 151-182.

Banerjee, Abhijit et al. (2015). "The Miracle of Microfinance? Evidence from a Randomized Evaluation". American Economic Journal: Applied Economics 7, 22-53.

Broderick, Tamara, Ryan Giordano, and Rachael Meager (2021). "An Automatic FiniteSample Robustness Metric: Can Dropping a Little Data Change Conclusions?" arXiv:2011.14999 [econ, stat]. arXiv: 2011.14999.

Brodeur, Abel, Nikolai Cook, and Anthony Heyes (2020). "Methods Matter: p-Hacking and Publication Bias in Causal Analysis in Economics". American Economic Review 110, 3634-3660.

LeBel, Etienne P. et al. (2013). "PsychDisclosure.org: Grassroots Support for Reforming Reporting Standards in Psychology". Perspectives on Psychological Science 8, 424-432.

Lenz, Gabriel S. and Alexander Sahn (2021). "Achieving Statistical Significance with Control Variables and Without Transparency". Political Analysis 29, 356-369.

Miguel, E. et al. (2014). "Promoting Transparency in Social Science Research". Science 343, $30-31$.

Ofosu, George K. and Daniel N. Posner (2021). "Pre-Analysis Plans: An Early Stocktaking". Perspectives on Politics, 1-17.

Simmons, Joseph P., Leif D. Nelson, and Uri Simonsohn (2011). "False-Positive Psychology: Undisclosed Flexibility in Data Collection and Analysis Allows Presenting Anything as Significant". Psychological Science 22, 1359-1366.

Stroebe, Wolfgang, Tom Postmes, and Russell Spears (2012). "Scientific Misconduct and the Myth of Self-Correction in Science". Perspectives on Psychological Science 7, 670-688.

Young, Alwyn (2020). "Consistency without Inference: Instrumental Variables in Practical Application". 